\title{
Cachexia in cancer patients
}

\begin{abstract}
Cancer cachexia (CC) is a multifactorial complex syndrome characterized by pathophysiological changes that alter body composition, quality of life, morbidity and mortality of cancer patients. CC is characterized by muscle depletion, with or without loss of adipose tissue, which cannot be completely reversed by current treatments. The pathogenesis of $\mathrm{CC}$ is multifactorial, including anorexia, metabolic alterations and increased muscle proteolysis. Despite the growing understanding of $\mathrm{CC}$, it is clinically under-recognized and frequently untreated. Identification of patients with pre-cachexia is of crucial importance for effective interventions. Integration of novel biological treatments with nutritional support and physical activity is a very promising strategy for multimodal anticancer therapies.
\end{abstract}

Volume I Issue I - 2017

\author{
CVanesa Díaz García, MTeresa Agulló \\ Ortuño \\ Laboratory of Translational Oncology, Health Research Institute \\ Hospital, Spain
}

\begin{abstract}
Correspondence: M Teresa Agulló-Ortuño, Laboratory of Translational Oncology, Health Research Institute Hospital 12 de Octubre (i+12), Avda de Córdoba S/N, 2804I Madrid, Spain, Email agullo@hI2o.es
\end{abstract}

Received: February 28, 2017 | Published: April 07, 2017

\section{Introduction}

Cancer is a leading cause of death worldwide. ${ }^{1}$ Besides, overall survival of cancer patients is negatively affected by the presence of comorbidities. In this context, more than one-half of all cancer patients suffer from cachexia. Cachexia is a debilitating condition with high incidence in cancer patients and may lead to delayed, missed, or decreased treatments. ${ }^{2}$ Cancer cachexia (CC) has been defined as a complex and multifactorial syndrome characterized by severe body weight loss, muscle depletion, with or without loss of adipose tissue, and increased protein catabolism, which cannot be completely reversed by available treatments and leads to progressive functional impairment. ${ }^{3,4}$ The origin of CC is related to several causes as tumor derived factors, host-derived factors inducing metabolic derangements or side effects of anticancer therapies, among others. Recently, a panel of international experts participated in a formal consensus process in order to develop a framework for the definition and the classification of cancer cachexia. ${ }^{3}$ The agreed diagnostic criteria for CC were a weight loss greater than $5 \%$, or a weight loss greater than $2 \%$ in patients already showing depletion according to their body mass index or skeletal muscle mass. ${ }^{3}$ Unfortunately, despite the significant advancement achieved during the last years, CC is still frequently under-recognized, untreated, and considered inevitable for cancer patients. ${ }^{2,5,6}$ In patients with cancer, a strong association exists between cachexia and poor quality of life, besides an increased morbidity and mortality. At present, it is estimated that about $20 \%$ of cancer patients die as a result of CC. ${ }^{7}$ In recent years, significant advances have been made in the knowledge of the pathogenic mechanisms underlying cachexia. However, such advances have not yet translated into effective strategies that can prevent or treat this devastating disorder which affect the quality of life of cancer patients. Nowadays, it is clear that multimodal strategies, physical activity, nutritional and metabolic interventions, and adequate pharmacological treatment are required to improve patients' outcomes. ${ }^{8}$ In such a context we have undertaken this review as an introduction and overview of current findings on classification and pathogenesis of $\mathrm{CC}$, and its implications on novel therapeutic strategies.

\section{Classification of cachexia}

In the last years, there has been a growing understanding of $\mathrm{CC}$ as a continuum that can progress through various stages: pre- cachexia, cachexia, and refractory cachexia. ${ }^{3,4,9} \mathrm{CC}$ ranges from nonsymptomatic inflammatory alterations with minimal muscle loss at early stage, to severe muscle wasting and low performance status in more advanced stages. Recent studies propose that cachexia should be considered a comorbidity of cancer, and highlighted the need to recognize the complete cachexia trajectory. ${ }^{8-10}$ These approaches have important implications since they might favor early recognition, diagnosis, and therapeutic interventions in cancer patients with cachexia. Early identification of patients with pre-cachexia would allow treatments that can prevent cachexia in these patients by timely appropriate and effective interventions. Unfortunately, those therapies have not been successful against cachexia. Thus, the early detection of nutritional impairments in cancer patients is absolutely necessary. ${ }^{9,11}$

\section{Pathogenesis of cancer cachexia}

Factors that contribute to cachexia are anorexia, defined as the reduction or loss of the desire to eat, and metabolic alterations, such as increased inflammatory status, impaired carbohydrate, protein and lipid metabolism, and increased muscle proteolysis. ${ }^{11}$ The pathogenesis of cancer-related anorexia is multifactorial and involves disruption of central and peripheral signals that physiologically regulate eating behavior. Increased resting energy expenditure, mostly secondary to the imbalance between pro-inflammatory and anti-inflammatory cytokines has been long considered a key factor in the pathogenesis of CC. ${ }^{12,13}$ However, this concept is controversial because of the observation that metabolic response in cancer may be extremely heterogeneous. ${ }^{14}$ Also, impaired glucose tolerance, insulin resistance and the increase in gluconeogenesis from amino acids and lactate, are frequently found during cancer disease. ${ }^{15}$ Nevertheless, there are no clear justifications for these alterations. In the course of CC, alterations in lipid metabolism are frequent, such as hyperlipidemia. ${ }^{13,16}$ Fatty acids and glycerol turnover is increased in cancer patients. The alterations in lipid metabolism are often detectable before the onset of cachexia and body weight loss. ${ }^{17,18}$ Among the mediators that may be involved in these metabolic disorders, insulin plays a prominent role. ${ }^{11}$

Modification of protein metabolism is also frequently in cancer patients, and includes increased acute phase proteins synthesis, increased total body proteins, alterations of plasma amino acid profile, and increased muscle wasting given that depletion of skeletal muscle is a relevant component of body weight loss in $\mathrm{CC} .{ }^{4}$ However, reduced 
protein anabolism in muscle may be involved as well. ${ }^{11}$ Malnutrition has been long recognized as a negative prognostic factor in cancer patients. Weight loss is an independent predictive factor of reduced survival, and has been associated with altered physical function, increased physical distress and impaired quality of life. Conversely, preventing weight loss positively influences survival and quality of life in patients undergoing cancer treatments. Moreover, muscle loss has been clearly identified as an independent predictor of mortality and reduced tolerance to treatment. ${ }^{19}$

\section{Therapeutic strategies for cancer cachexia}

Treatment of CC might ameliorate life expectancy, improving body composition and quality of life to maximize survival in these patients..$^{20} \mathrm{An}$ adequate nutritional support aimed at satisfying protein and caloric needs of cancer patients is mandatory, given that nutrient intake in these patients is frequently suboptimum. ${ }^{2} 1$ Recent studies have revealed that systemic inflammation has a relevant role in determining the metabolic derangements leading to loss of muscle. ${ }^{13,20}$ Thereby, clinical trials in CC have been mainly focused on appetite stimulants, non-steroidal anti-inflammatory drugs, anabolic agents, etc.

Muscaritoli et al. ${ }^{11}$ have proposed the "parallel pathway" as a preventative and therapeutic tool for $\mathrm{CC}$, aimed at the early recognition, prevention and treatment of the metabolic and nutritional derangements occurring in cancer. Their proposal involve a multiprofessional and multimodal approach: since the diagnosis of cancer is performed, patients should be constantly monitored by nutritionists in parallel with the oncologists, to early assess nutritional status and to diagnose metabolic modifications. These approaches may positively affect quality of life and tolerance to antineoplastic treatments, supporting the view that preventative metabolic and nutritional interventions should become an integral part of the multimodal anticancer treatments.

According to the newest knowledge in CC, novel therapies mainly aimed at acting on specific molecular targets such as myostatin, ghrelin, IL-6, IL-1, and skeletal muscle androgen receptor has been proposed. However, the only available intervention is nowadays represented by a multimodal approach aimed at counteracting the development of cancer cachexia. In this light, Muscaritoli et al. ${ }^{9}$ propose the T.A.R.G.E.T. approach, encompassing both active actions and research within different domains, namely Teaching, Awareness, Recognition, Genetics, Exercise/Early intervention and Treatment of CC.

Temel et al..$^{22}$ have recently shown the results of two phase III trials (ROMANA 1 and ROMANA 2) assessing the effects of anamorelin, an oral ghrelin-receptor agonist, on CC in patients with advanced nonsmall-cell lung cancer receiving antitumoral treatment. The finding that this drug might attenuate the loss of muscle mass and cachexia related symptom during treatment might have major implications in clinical practice. These trials offer new hopes for more effective therapeutic strategies for $\mathrm{CC}$, where the combination of drug treatment, exercise, and proper nutrition should be integrated to improve quality of life and clinical outcomes in these patients.

Also, it have been shown that specialized nutritional support enriched with fish-oil n-3 polyunsaturated fatty acids and micronutrients supplementation may positively affect quality of life, performance status and physical activity in patients with different cancer as lung, colorectal and esophageal cancer. ${ }^{23-25}$ There are some controversy on these substrates efficacy, but their anti-inflammatory, immunomodulating and restoring properties in CC appear useful. ${ }^{9,26}$ Pro-inflammatory cytokines such as TNF- $\alpha$ and IL-6 are direct mediators of sarcopenia, while additional inflammatory processes have been shown to maintain the wasting process in cachexia. Hong et al., have recently proposed a novel therapeutically approach against inflammation. They administered, in patients with advanced cancer and cachexia, an intravenous anti-IL-1 monoclonal antibody, and documented after 8 weeks a decrease of median plasma IL-6 concentrations and a significant increase of lean body mass. ${ }^{27}$

The effects of myostatin inhibition in sarcopenia patients has been shown in a randomized, phase II trial study testing the effects of an anti-myostatin humanized monoclonal antibody in older adults who recently reported falls and low muscle strength and power. Patients receiving this treatment, showed an increase in lean mass and a significant improvement of muscle power expressed as improved stair-climbing time, fast gait speed, and chair rise with arms. ${ }^{28}$

Since androgens increase muscle mass and strength, its use in cancer patients presenting muscle wasting have been proposed. ${ }^{29}$ Enobosarm is a novel nonsteroidal selective androgen receptor's modulator. Phase II, and III clinical trials (POWER1 and POWER2) of enobosarm documented an increase in lean body mass and improvement in physical functions in cancer patients presenting muscle wasting. ${ }^{30,31}$ The better understanding of some of the mechanisms involved in the pathogenesis of $\mathrm{CC}$ is progressively allowing for the identification of several potential molecular targets for interventions with biological therapies. However, no single pharmacological agent shows a clear efficacy in counteracting CC. A randomized phase III clinical trial of 5 different arms of treatment, showed that the combination of a progestational agent, EPA-enriched nutritional supplement, L-carnitine and thalidomide is more effective than each single treatment alone. ${ }^{32}$ The multimodal treatment efficacy was also evaluated in a recent study assessing the ability of L-carnitine, celecoxib and megestrol acetate in improving total daily physical activity, CC symptoms, and functional status as well as increasing lean body mass. ${ }^{33}$ Thus, multimodal intervention should be scheduled in parallel with anti-cancer therapies and may consist in nutritional intervention, exercise and rehabilitation program, and pharmacological interventions.

On the other hand, in malnourished cancer patients undergoing surgery, artificial nutritional support is often indicated. The rationale is based on the assumption that malnourished patients with cancer are at high risk for postoperative complications, and that cancerrelated malnutrition can be reverted through nutritional support, also reducing surgical risk. However, there are some ethical and nutritional aspects that must be taken into account, especially in terminal cancer patients. ${ }^{34}$

\section{Conclusion}

$\mathrm{CC}$ is still under-recognized, prevented and inadequately treated in cancer patients, in spite of its severe consequences on prognosis and quality of life of patients. Many authors agree that CC should be considered a comorbidity of cancer. However, the perception and clinical practices concerning to CC vary among clinicians worldwide. ${ }^{6}$ On the other hand, the lack of standardized tools for identify patients who have symptoms of $\mathrm{CC}$, is one factor leading to suboptimal CC management. ${ }^{35}$ Unfortunately, the identification and treatment of cachexia frequently arrive in a late stage, which renders integrated treatments less effective. 
In this perspective, a novel nutritional and metabolic approach to cancer patients appears mandatory. The strong impact that CC has on cancer patients' outcome and quality of life suggests that nutritional issues should be taken into consideration from the beginning of the symptoms of the disease. Therefore, cancer patients should be nutritionally monitored in parallel with the oncologist by a multidisciplinary team.

The complex and multifactorial pathogenesis of cancer related metabolic and nutritional impairment requires multimodal preventative and therapeutic strategies for their prevention or attenuation and treatment. Integration of novel biological treatments with metabolic and nutritional support appears to be the most scientifically sound and promising pathway to the future of cancer supportive therapies. This view has clinical implications, since it might favor early recognition, diagnosis and therapeutic interventions for $\mathrm{CC}$, positively impacting on survival and quality of life in these patients.

\section{Acknowledgements}

None.

\section{Conflict of interest}

The author declares no conflict of interest.

\section{References}

1. Torre LA, Siegel RL, Ward EM, et al. Global cancer incidence and mortality rates and trends-an update. Cancer Epidemiol Biomarkers Prev. 2016;25(1):16-27.

2. Sun L, Quan XQ, Yu S. An epidemiological survey of cachexia in advanced cancer patients and analysis on its diagnostic and treatment status. Nutr Cancer. 2015;67(7):1056-1062.

3. Fearon K, Strasser F, Anker SD, et al. Definition and classification of cancer cachexia: an international consensus. Lancet Oncol. 2011;12(5):489-495.

4. Muscaritoli M, Anker SD, Argilé J, et al. Consensus definition of sarcopenia, cachexia and pre-cachexia: joint document elaborated by Special Interest Groups (SIG) "cachexia-anorexia in chronic wasting diseases" and "nutrition in geriatrics". Clin Nutr. 2010;29(2):154-159.

5. Vanchieri C. Cachexia in cancer: is it treatable at the molecular level. $J$ Natl Cancer Inst. 2010;102(22):1694-1697.

6. Muscaritoli M, Rossi Fanelli F, Molfino A. Perspectives of health care professionals on cancer cachexia: results from three global surveys. Ann Oncol. 2016;27(12):2230-2236.

7. Argilés JM, Busquets S, Stemmler B, et al. Cancer cachexia: understanding the molecular basis. Nat Rev Cancer. 2014;14(11):754762.

8. Gagnon B, Murphy J, Eades M, et al. A prospective evaluation of an interdisciplinary nutrition-rehabilitation program for patients with advanced cancer. Curr Oncol. 2013;20(6):310-318.

9. Muscaritoli M, Molfino A, Lucia S, et al. Cachexia: A preventable comorbidity of cancer. A T.A.R.G.E.T. approach. Crit Rev Oncol Hematol. 2015;94(2):251-259.

10. Blum D, Stene GB, Solheim TS, et al. Validation of the consensusdefinition for cancer cachexia and evaluation of a classification model-a study based on data from an international multicentre project (EPCRCCSA). Ann Oncol. 2014;25(8):1635-1642.

11. Muscaritoli M, Molfino A, Gioia G, et al. The "parallel pathway": a novel nutritional and metabolic approach to cancer patients. Intern Emerg Med. 2011;6(2):105-112.
12. Patel HJ, Patel BM. TNF- $\alpha$ and cancer cachexia: Molecular insights and clinical implications. Life Sci. 2017;170:56-63.

13. Batista JL Jr, Peres SB, MacDonald ME, et al. Adipose tissue inflammation and cáncer cachexia: posible role of nuclear transcription factors. Cytokine. 2012;57(1):9-16.

14. Porporato PE. Understanding cachexia as a cancer metabolism syndrome. Oncogenesis. 2016;5(2):e200.

15. Wagner EF, Petruzzelli M. Cancer metabolism: A waste of insulin interference. Nature. 2015;521(7553):430-431.

16. Busquets S, Carbó N, Almendro V, et al. Hyperlipemia: a role in regulating UCP3 gene expression in skeletal muscle during cancer cachexia. FEBS Lett. 2001;505:255-258.

17. Saavedra García P, Nichols K, Mahmud Z, et al. Unravelling the role of fatty acid metabolism in cancer through the FOXO3-FOXM1 axis. Mol Cell Endocrinol. 2017;7207(17):30016-30030.

18. Korber J, Pricelius S, Heidrich M, et al. Increased lipid utilization in weight losing and weight stable cancer patients with normal body weight. Eur J clin Nutr. 1999;53(9):540-545.

19. Drescher C, Konishi M, Ebner N, et al. Loss of muscle mass: current developments in cachexia and sarcopenia focused on biomarkers and treatment. J Cachexia Sarcopenia Muscle. 2015;6(4):303-311.

20. Molfino A, Amabile MI, Rossi Fanelli F, et al. Novel therapeutic options for cachexia and sarcopenia. Expert Opin Biol Ther. 2016;16(10):12391244.

21. Prado CM, Sawyer MB, Ghosh S, et al. Central tenet of cancer cachexia therapy: do patients with advanced cancer have exploitable anabolic potential. Am J Clin Nutr. 2013;98:1012-1019.

22. Temel JS, Abernethy AP, Currow DC, et al. Anamorelin in patients with non-small-cell lung cancer and cachexia (ROMANA 1 and ROMANA 2): results from two randomised, double-blind, phase 3 trials. Lancet Oncol. 2016;17(4):519-531.

23. van der Meij BS, Langius JA, Spreeuwenberg MD, et al. Oral nutritional supplements containing n-3 polyunsaturated fatty acids affect quality of life and functional status in lung cancer patients during multimodality treatment: an RCT. Eur J Clin Nutr. 2012;66(3):399-404.

24. Read JA, Beale PJ, Volker DH, et al. Nutrition intervention using an eicosapentaenoic acid (EPA)-containing supplement in patients with advanced colorectal cancer. Effects on nutritional and inflammatory status: a phase II trial. Support Care Cancer. 2007;15(3):301-307.

25. Ryan AM, Reynolds JV, Healy L, et al. Enteral nutrition enriched with eicosapentaenoic acid (EPA) preserves lean body mass following esophageal cancer surgery: results of a double-blinded randomized controlled trial. Ann Surg. 2009;249(3):355-363.

26. Gullett NP, Mazurak VC, Hebbar G, et al. Nutritional interventions for cancer-induced cachexia. Curr Probl Cancer. 2011;35(2):58-90.

27. Hong DS, Hui D, Bruera E, et al. MABp1, a first-in-class true human antibody targeting IL-1a in refractory cancers: an open-label, phase 1 dose-escalation and expansion study. Lancet Oncol. 2014;15(6):656666.

28. Becker C, Lord SR, Studenski SA, et al. Myostatin antibody (LY2495655) in older weak fallers: a proof-of-concept, randomised, phase 2 trial. Lancet Diabetes Endocrinol. 2015;3(12):948-957.

29. Dubois V, Laurent M, Boonen S, et al. Androgens and skeletal muscle: cellular and molecular action mechanisms underlying the anabolic actions. Cell Mol Life Sci. 2012;69:1651-1667.

30. Dobs AS, Boccia RV, Croot CC, et al. Effects of enobosarm on muscle wasting and physical function in patients with cancer: a double-blind, randomised controlled phase 2 trial. Lancet Oncol. 2013;14(4):335-345. 
31. Srinath R, Dobs A. Enobosarm (GTx-024, S-22): a potential treatment for cachexia. Future Oncol. 2014;10(2):187-194.

32. Mantovani G, Macciò A, Madeddu C, et al. Randomized phase III clinical trial of five different arms of treatment in 332 patients with cancer cachexia. Oncologist. 2010;15(2):200-211.

33. Madeddu C, Dessì M, Panzone F, et al. Randomized phase III clinical trial of a combined treatment with carnitine + celecoxib \pm megestrolacetate for patients with cancer-related anorexia/cachexia syndrome. Clin Nutr. 2012;31(2):176-182.
34. Bozzetti F, Arends J, Lundholm K, et al. ESPEN Guidelines on parenteral nutrition: non-surgical oncology. Clin Nutr. 2009;28(4):445-454.

35. Del Fabbro E, Jatoi A, Davis, et al. Health professionals' attitudes toward the detection and management of cancer-related anorexia-cachexia syndrome, and a proposal for standardized assessment. J Community Support Oncol. 2015;13:181-187. 